\title{
CONJECTURES OF BRUMER, GROSS AND STARK
}

\author{
ANDREAS NICKEL
}

\begin{abstract}
We give an introduction to generalisations of conjectures of Brumer and Stark on the annihilator of the class group of a number field. We review the relation to the equivariant Tamagawa number conjecture, the main conjecture of Iwasawa theory for totally real fields, and a conjecture of Gross on the behaviour of $p$-adic Artin $L$-functions at zero.
\end{abstract}

\section{INTRODUCTION}

Let $L / K$ be a finite Galois extension of number fields with Galois group $G$. To each finite set $S$ of places of $K$ containing all the archimedean places, one can associate a so-called 'Stickelberger element' $\theta_{S}$ in the centre of the group algebra $\mathbb{C}[G]$. This element is constructed from values at $s=0$ of the $S$-truncated Artin $L$-series attached to the complex irreducible characters of $G$. In particular, $\theta_{S}$ is analytic in nature. By a result of Siegel [34] one knows that $\theta_{S}$ always has rational coefficients.

Let $\mu_{L}$ and $\mathrm{cl}_{L}$ be the roots of unity and the class group of $L$, respectively. Suppose that $S$ also contains all places of $K$ which ramify in $L / K$. Then it was independently shown by Cassou-Noguès [8], Deligne-Ribet [13] and Barsky [1] that for abelian $G$ one has

$$
\operatorname{Ann}_{\mathbb{Z}[G]}\left(\mu_{L}\right) \theta_{S} \subseteq \mathbb{Z}[G]
$$

where $\operatorname{Ann}_{R}(M)$ denotes the annihilator ideal of $M$ regarded as a module over the ring $R$. In other words, the coefficients of $\theta_{S}$ are almost integral. Now Brumer's conjecture simply asserts that $\operatorname{Ann}_{\mathbb{Z}[G]}\left(\mu_{L}\right) \theta_{S}$ annihilates $\mathrm{cl}_{L}$. In the case $K=\mathbb{Q}$ Brumer's conjecture is just Stickelberger's theorem from the late 19th century [36]. Roughly speaking, the conjecture predicts that an analytic object gives constraints on the structure of an arithmetic object. It is this kind of conjecture which is often called a 'Stark-type conjecture'.

In fact, Harold Stark suggested the following refinement of Brumer's conjecture. Let $w_{L}$ be the cardinality of $\mu_{L}$ and fix a fractional ideal $\mathfrak{a}$ in $L$. We will denote the action of $G$ on $\mathfrak{a}$ and on its class in $\mathrm{cl}_{L}$ by exponents on the right as usual. Then the BrumerStark conjecture not only predicts that $\mathfrak{a}^{w_{L} \theta_{S}}$ becomes principal, but also gives precise information about a generator of that ideal.

In this survey article we explain recent generalisations of these conjectures to arbitrary, not necessarily abelian Galois extensions; these are due to the author [27] and, independently and in even greater generality, due to Burns [6]. A further, slightly different approach has been developed by Dejou and Roblot [14].

We discuss the relation to further conjectures in the field such as the equivariant Tamagawa number conjecture of Burns and Flach [3] and the main conjecture of equivariant Iwasawa theory which (under a suitable condition) has been proven by Ritter-Weiss [33]

Date: Version of 10th October 2017.

2010 Mathematics Subject Classification. 11R42, 11R29, 11R23.

Key words and phrases. Stark conjectures, Brumer's conjecture, $L$-values, class groups, equivariant Tamagawa number conjecture, main conjecture, Iwasawa theory. 
and, independently, by Kakde [25]. A conjecture of Gross [15] on the behaviour of $p$-adic Artin $L$-series at $s=0$ also plays a pivotal role.

Roughly speaking, the latter conjecture asserts that (i) the order of vanishing at $s=0$ of the $p$-adic $L$-series coincides with the order of vanishing at $s=0$ of a corresponding complex $L$-series and (ii) the special values of the $p$-adic and the complex $L$-series at $s=0$ coincide up to some explicit $p$-adic regulator. Considerable progress has been made in the recent years by Spiess [35] and Burns [7] on part (i), and by Dasgupta, Kakde and Ventullo [12] on part (ii). We also explain their results and the relation to the equivariant Tamagawa number conjecture due to Burns [7] as well as the relation to the (non-abelian) Brumer-Stark conjecture due to Johnston and the author [23].

We provide no proofs unless they are short and we feel that it might help for a better understanding. Instead we include some examples to illustrate occurring obstacles and the underlying ideas how to overcome them.

Notation and conventions. All rings are assumed to have an identity element and all modules are assumed to be left modules unless otherwise stated. Unadorned tensor products will always denote tensor products over $\mathbb{Z}$. We fix the following notation:

$\begin{array}{ll}R^{\times} & \text {the group of units of a ring } R \\ \zeta(R) & \text { the centre of a ring } R \\ \operatorname{Ann}_{R}(M) & \text { the annihilator ideal of the } R \text {-module } M \\ M_{m \times n}(R) & \text { the set of all } m \times n \text { matrices with entries in a ring } R \\ K_{\infty} & \text { the cyclotomic } \mathbb{Z}_{p} \text {-extension of the number field } K \\ \mu_{K} & \text { the roots of unity of a field } K \\ \mathrm{cl}_{K} & \text { the class group of a number field } K \\ K^{c} & \text { an algebraic closure of a field } K \\ \operatorname{Irr}_{F}(G) & \text { the set of } F \text {-irreducible characters of the (pro)-finite group } G \\ & \text { (with open kernel) where } F \text { is a field of characteristic } 0\end{array}$

\section{Preliminaries}

1.1. Ray class groups. Let $L / K$ be a finite Galois extension of number fields with Galois group $G$. For each place $v$ of $K$ we fix a place $w$ of $L$ above $v$ and write $G_{w}$ and $I_{w}$ for the decomposition group and inertia subgroup of $L / K$ at $w$, respectively. When $w$ is a finite place, we choose a lift $\phi_{w} \in G_{w}$ of the Frobenius automorphism at $w$; moreover, we write $\mathfrak{P}_{w}$ for the associated prime ideal in $L$ and $|\cdot|_{w}$ for the corresponding absolute value. We denote the cardinality of the residue field of $K$ at $v$ by $N(v)$.

For any set $S$ of places of $K$, we write $S(L)$ for the set of places of $L$ which lie above those in $S$. Now let $S$ be a finite set of places of $K$ containing the set $S_{\infty}=S_{\infty}(K)$ of archimedean places and let $T$ be a second finite set of places of $K$ such that $S \cap T=\emptyset$. We write $\mathrm{cl}_{L}^{T}$ for the ray class group of $L$ associated to the ray $\mathfrak{M}_{L}^{T}:=\prod_{w \in T(L)} \mathfrak{P}_{w}$ and $\mathcal{O}_{L, S}$ for the ring of $S(L)$-integers in $L$. Let $\mathcal{O}_{L}:=\mathcal{O}_{L, S_{\infty}}$ be the ring of integers in $L$. Let $S_{f}$ be the set of all finite places in $S$; then there is a natural map $\mathbb{Z}\left[S_{f}(L)\right] \rightarrow \mathrm{cl}_{L}^{T}$ which sends each place $w \in S_{f}(L)$ to the corresponding class $\left[\mathfrak{P}_{w}\right] \in \mathrm{cl}_{L}^{T}$. We denote the cokernel of this map by $\mathrm{cl}_{L, S}^{T}$. When $T$ is empty, we abbreviate $\mathrm{cl}_{L, S}^{\emptyset}$ to $\mathrm{cl}_{L, S}$ so that in particular $\mathrm{cl}_{L, \emptyset}=\mathrm{cl}_{L}$ is the usual class group of $L$. Moreover, we denote the $S(L)$-units of $L$ by $E_{L, S}$ and define $E_{L, S}^{T}:=\left\{x \in E_{L, S}: x \equiv 1 \bmod \mathfrak{M}_{L}^{T}\right\}$. All these modules are equipped with a natural $G$-action and we have an exact sequence of $\mathbb{Z}[G]$-modules

$$
0 \longrightarrow E_{L, S}^{T} \longrightarrow E_{L, S} \longrightarrow\left(\mathcal{O}_{L, S} / \mathfrak{M}_{L}^{T}\right)^{\times} \stackrel{\nu}{\longrightarrow} \mathrm{cl}_{L, S}^{T} \longrightarrow \mathrm{cl}_{L, S} \longrightarrow 0
$$


where the map $\nu$ lifts an element $\bar{x} \in\left(\mathcal{O}_{L, S} / \mathfrak{M}_{L}^{T}\right)^{\times}$to $x \in \mathcal{O}_{L, S}$ and sends it to the ideal class $[(x)] \in \mathrm{cl}_{L, S}^{T}$ of the principal ideal $(x)$.

1.2. Equivariant Artin $L$-values. Let $S$ be a finite set of places of $K$ containing $S_{\infty}$. Let $\operatorname{Irr}_{\mathbb{C}}(G)$ denote the set of complex irreducible characters of $G$. For $\chi \in \operatorname{Irr}_{\mathbb{C}}(G)$, let $V_{\chi}$ be a left $\mathbb{C}[G]$-module with character $\chi$. We write $L_{S}(s, \chi)$ for the $S$-truncated Artin $L$-function attached to $\chi$ which for $\operatorname{Re}(s)>1$ is given by the Euler product

$$
L_{S}(s, \chi)=\prod_{v \notin S} \operatorname{det}\left(1-N(v)^{-s} \phi_{w} \mid V_{\chi}^{I_{w}}\right)^{-1} .
$$

Each element in $\mathbb{C}[G]$ may be viewed as a complex valued function on $G$. The irreducible characters constitute a basis of the centre and we thus have a canonical isomorphism $\zeta(\mathbb{C}[G]) \simeq \prod_{\chi \in \operatorname{Irr}_{\mathbb{C}}(G)} \mathbb{C}$. We define the equivariant $S$-truncated Artin $L$-function to be the meromorphic $\zeta(\mathbb{C}[G])$-valued function

$$
L_{S}(s):=\left(L_{S}(s, \chi)\right)_{\chi \in \operatorname{Irr}_{\mathbb{C}}(G)} .
$$

If $T$ is a second finite set of places of $K$ such that $S \cap T=\emptyset$, we define

$$
\delta_{T}(s, \chi)=\prod_{v \in T} \operatorname{det}\left(1-N(v)^{1-s} \phi_{w}^{-1} \mid V_{\chi}^{I_{w}}\right)
$$

and

$$
\delta_{T}(s):=\left(\delta_{T}(s, \chi)\right)_{\chi \in \operatorname{Irr}_{\mathbb{C}}(G)} .
$$

We set

$$
\Theta_{S, T}(s):=\delta_{T}(s) \cdot L_{S}(s)^{\sharp},
$$

where ${ }^{\sharp}: \mathbb{C}[G] \rightarrow \mathbb{C}[G]$ denotes the anti-involution induced by $g \mapsto g^{-1}$ for $g \in G$. Note that $L_{S}(s)^{\sharp}=\left(L_{S}(s, \check{\chi})\right)_{\chi \in \operatorname{Irr} C}(G)$ where $\check{\chi}$ denotes the character contragredient to $\chi$. The functions $\Theta_{S, T}(s)$ are the so-called $(S, T)$-modified $G$-equivariant $L$-functions, and we define Stickelberger elements

$$
\theta_{S}^{T}(L / K)=\theta_{S}^{T}:=\Theta_{S, T}(0) \in \zeta(\mathbb{Q}[G]) .
$$

Note that a priori we only have $\theta_{S}^{T} \in \zeta(\mathbb{C}[G])$, but by a result of Siegel [34] we know that $\theta_{S}^{T}$ has rational coefficients. If $T$ is empty, we abbreviate $\theta_{S}^{T}$ to $\theta_{S}$.

Remark 1.1. Let $\chi \in \operatorname{Irr}_{\mathbb{C}}(G)$ and let $r_{S}(\chi)$ be the order of vanishing of $L_{S}(s, \chi)$ at $s=0$. Then by [37, Chapitre I, Proposition 3.4] one has

$$
r_{S}(\chi)=\left(\sum_{v \in S} \operatorname{dim}_{\mathbb{C}}\left(V_{\chi}^{G_{w}}\right)\right)-\operatorname{dim}_{\mathbb{C}}\left(V_{\chi}^{G}\right) .
$$

Thus if either $\chi$ is non-trivial and $S$ contains an (infinite) place $v$ such that $V_{\chi}^{G_{w}} \neq 0$ or $\chi$ is trivial and $|S|>1$ then the $\chi$-part of $\theta_{S}^{T}$ vanishes. Now suppose that $S$ contains all ramified primes. Then if $\theta_{S}^{T}$ is non-trivial, precisely one of the following possibilities occurs: (i) $K$ is totally real and $L$ is totally complex, (ii) $K$ is an imaginary quadratic field, $L / K$ is unramified and $S=S_{\infty}$ or (iii) $L=K=\mathbb{Q}$ and $S=S_{\infty}$. 


\section{THE ABELIAN CASE}

In this section we assume that the extension $L / K$ is abelian. Let $\mu_{L}$ denote the roots of unity in $L$. It was independently shown in [1, 8, 13] that one has

$$
\operatorname{Ann}_{\mathbb{Z}[G]}\left(\mu_{L}\right) \theta_{S} \subseteq \mathbb{Z}[G]
$$

whenever $S$ contains the set $S_{\text {ram }}$ of all places of $K$ that ramify in $L / K$. We now state Brumer's conjecture as discussed by Tate [37.

Conjecture $2.1(B(L / K, S))$. Let $L / K$ be an abelian extension of number fields with Galois group $G$ and let $S$ be a finite set of places of $K$ containing the set $S_{\infty}$ and all places of $K$ that ramify in $L / K$. Then one has

$$
\operatorname{Ann}_{\mathbb{Z}[G]}\left(\mu_{L}\right) \theta_{S} \subseteq \operatorname{Ann}_{\mathbb{Z}[G]}\left(\mathrm{cl}_{L}\right) .
$$

We will discuss relevant results on Brumer's conjecture and its generalisations in $\$ 4$ below. Here we only mention that for absolutely abelian number fields Brumer's conjecture follows from Stickelberger's theorem from the late 19th century.

Theorem 2.2 (Stickelberger [36]). Assume that $L$ is abelian over $\mathbb{Q}$. Then, Brumer's conjecture $B(L / K, S)$ holds.

Remark 2.3. Consider the three cases of Remark 1.1. In case (iii), Brumer's conjecture (and also the Brumer-Stark conjecture below) is trivial. Case (ii) follows from the fact that each ideal of $L$ becomes principal in the Hilbert class field of $L$ (see [18, Remark 6.3]). Finally, case (i) can be reduced to the case that $L$ is a CM-field (see [18, Proposition $6.4])$.

Therefore, we shall henceforth assume that $L / K$ is a CM-extension, that is, $L$ is a CM-field, $K$ is totally real and complex conjugation induces a unique automorphism $j$ in $G$.

Let $w_{L}$ be the cardinality of $\mu_{L}$. Then clearly $w_{L} \in \operatorname{Ann}_{\mathbb{Z}[G]}\left(\mu_{L}\right)$ and so Brumer's conjecture asserts that $\mathfrak{a}^{w_{L} \theta_{S}}$ is a principal ideal for every fractional ideal $\mathfrak{a}$ in $L$. The Brumer-Stark conjecture now gives precise information on a generator of this ideal. To make this precise, we need the following definition.

Definition 2.4. Let $L$ be a CM-field. Then, $\epsilon \in L^{\times}$is called an anti-unit if $\epsilon^{1+j}=1$.

Conjecture $2.5(B S(L / K, S))$. Let $L / K$ be an abelian $C M$-extension with Galois group $G$ and let $S$ be a finite set of places of $K$ containing the set $S_{\infty}$ and all places of $K$ that ramify in $L / K$. Then, for every non-zero fractional ideal $\mathfrak{a}$ in $L$, there is an anti-unit $\epsilon \in L^{\times}$such that

(i) $\mathfrak{a}^{w_{L} \theta_{S}}=(\epsilon)$.

(ii) The extension $L\left(w_{E} \in \bar{\epsilon}\right) / K$ is abelian.

Here, the first part almost follows from $B(L / K, S)$, and Harold Stark originally suggested that the second condition might hold. The conjecture was first stated in published form by Tate [37].

Remark 2.6. An anti-unit $\epsilon \in L^{\times}$that fulfils (1) is determined up to a root of unity.

In order to generalise these conjectures to arbitrary Galois CM-extensions, we need reformulations of both conjectures. For this, we introduce the following terminology. Let $S$ and $T$ be two finite sets of places of $K$. We then say that $\operatorname{Hyp}(S, T)$ is satisfied if the following holds: 
- $S$ contains all the archimedean places of $K$ and all places which ramify in $L / K$, i.e. $S \supseteq S_{\text {ram }} \cup S_{\infty}$.

- $S \cap T=\emptyset$.

- $E_{L, S}^{T}$ is torsionfree.

Remark 2.7. Note that $E_{L, S}^{T}$ is torsionfree whenever $T$ contains at least two places of different residue characteristic or at least one place of sufficiently large norm.

Lemma 2.8. Let $S$ be a finite set of places of $K$ containing $S_{\text {ram }} \cup S_{\infty}$. Then, the elements $1-N(v) \phi_{w}^{-1}$, where $v$ runs through all the finite places of $K$ such that the sets $S$ and $T_{v}:=\{v\}$ satisfy $\operatorname{Hyp}\left(S, T_{v}\right)$, generate $\operatorname{Ann}_{\mathbb{Z}[G]}\left(\mu_{L}\right)$. Moreover, if we restrict to totally decomposed primes $v$, the greatest common divisor of the integers $1-N(v)$ equals $w_{L}$.

Proof. This is an obvious reformulation of [37, Ch. IV, Lemma 1.1].

Corollary 2.9. Let $L / K$ be an abelian extension of number fields and let $S$ be a finite set of places of $K$ containing the set $S_{\infty}$ and all places of $K$ that ramify in $L / K$. Then, Brumer's conjecture $B(L / K, S)$ holds if and only if

$$
\theta_{S}^{T} \in \operatorname{Ann}_{\mathbb{Z}[G]}\left(\mathrm{cl}_{L}\right)
$$

for all finite sets $T$ of $L$ such that $\operatorname{Hyp}(S, T)$ is satisfied.

Proof. We have $\theta_{S}^{T}=\delta_{T}(0) \theta_{S}$ and $\delta_{T}(0) \in \operatorname{Ann}_{\mathbb{Z}[G]}\left(\mu_{L}\right)$ whenever $\operatorname{Hyp}(S, T)$ is satisfied. As $\delta_{T_{v}}(0)=1-N(v) \phi_{w}^{-1}$, the result follows from Lemma 2.8 .

Let $N_{L / K}: L^{\times} \rightarrow K^{\times}$be the field-theoretic norm map. For $\epsilon \in L^{\times}$we define

$$
S_{\epsilon}:=\left\{v \text { finite place of } K: v \mid N_{L / K}(\epsilon)\right\} .
$$

Proposition 2.10. Let $S$ be a finite set of places of $K$ containing $S_{\text {ram }} \cup S_{\infty}$. Then, the Brumer-Stark conjecture $B S(L / K, S)$ is equivalent to the following claim: For every non-zero fractional ideal $\mathfrak{a}$ of $L$ there is an anti-unit $\epsilon \in L^{\times}$such that

$$
\mathfrak{a}^{w_{L} \cdot \theta_{S}}=(\epsilon)
$$

and, for each finite set $T$ of primes of $K$ such that $\operatorname{Hyp}\left(S \cup S_{\epsilon}, T\right)$ is satisfied, there is an $\epsilon_{T} \in E_{S_{\epsilon}}^{T}$ such that

$$
\epsilon^{\delta_{T}(0)}=\epsilon_{T}^{w_{L}}
$$

Proof. This is [37, Ch. IV, Prop. 1.2].

Remark 2.11. In many cases, one can omit the condition that $\epsilon$ is an anti-unit: Suppose that the order of $\mathfrak{a}$ in the class group is odd. Then, we may write $\mathfrak{a}=\mathfrak{b}^{2}(u)$ for some $u \in L^{\times}$. Now assume that $\mathfrak{b}^{w_{L} \theta_{S}}$ is principal and generated by some $\beta \in L^{\times}$such that (2.2) holds with $\epsilon$ replaced with $\beta$. As $(1-j) \theta_{S}=2 \theta_{S}$, we then have $\mathfrak{a}^{w_{L} \theta_{S}}=(\epsilon)$, where $\epsilon:=u^{w_{L} \theta_{S}} \cdot \beta^{1-j}$ is an appropriate anti-unit.

\section{The GENERAL CASE}

3.1. How to generalise to non-abelian extensions? Now let $L / K$ be an arbitrary Galois CM-extension with Galois group $G$. How can we formulate Brumer's conjecture and the Brumer-Stark conjecture for this more general situation?

We still have the Stickelberger elements $\theta_{S}^{T}$ at our disposal. In view of Corollary 2.9 one is tempted to conjecture that $\theta_{S}^{T}$ still annihilates the class group whenever $\operatorname{Hyp}(S, T)$ 
is satisfied. Although the Stickelberger elements always belong to the centre of $\mathbb{Q}[G]$, it is, however, in general not true that $\theta_{S}^{T}$ has integral coefficients. Thus $\theta_{S}^{T}$ does in general not even act on the class group!

Example 3.1. This example is due to Nomura [32, §6]. Let $\alpha$ be a root of $x^{3}-11 x+7$ and set $L=\mathbb{Q}(\sqrt{-3}, \sqrt{4001}, \alpha)$. Then, $L / \mathbb{Q}$ is a Galois CM-extension with Galois group $G$ isomorphic to $\mathbb{Z} / 2 \mathbb{Z} \times S_{3}$, where $j$ generates the first factor and $S_{3}$ denotes the symmetric group on 3 letters. We write

$$
S_{3}=\left\langle\sigma, \tau \mid \sigma^{3}=\tau^{2}=1, \tau \sigma \tau^{-1}=\sigma^{-1}\right\rangle .
$$

Then $S_{\text {ram }}=\{3,4001\}$ and for $S=S_{\text {ram }} \cup S_{\infty}$ and $T=\{7\}$ one has

$$
\theta_{S}^{T}=\frac{1}{3}(1-j)\left(3410-1774\left(\sigma+\sigma^{2}\right)+44\left(\tau+\sigma \tau+\sigma^{2} \tau\right)\right)
$$

which visibly does not belong to $\mathbb{Z}[G]$.

The idea is to replace the centre of $\mathbb{Z}[G]$ by a larger ring $\mathcal{I}(G)$ such that $\theta_{S}^{T}$ always belongs to $\mathcal{I}(G)$ and such that $\mathcal{I}(G)=\mathbb{Z}[G]$ when $G$ is abelian. In order to achieve annihilators, one then has to multiply by a certain 'denominator ideal' $\mathcal{H}(G)$. We next introduce these purely algebraic objects.

3.2. Denominator ideals and the integrality ring. Let $R$ be a Noetherian integrally closed domain with field of fractions $E$. Let $A$ be a finite-dimensional separable $E$ algebra and let $\mathfrak{A}$ be an $R$-order in $A$. Our main examples are group rings $\mathfrak{A}=R[G]$ and $A=E[G]$, where $R$ and $E$ are either $\mathbb{Z}$ and $\mathbb{Q}$ or $\mathbb{Z}_{p}$ and $\mathbb{Q}_{p}$ for a prime $p$, respectively. The reduced norm map $\mathrm{nr}=\mathrm{nr}_{A}: A \longrightarrow \zeta(A)$ is defined component-wise on the Wedderburn decomposition of $A$ and extends to matrix rings over $A$ (see [10, $\S 7 \mathrm{D}]$ ). We choose a maximal $R$-order $\mathfrak{M}$ such that $\mathfrak{A} \subseteq \mathfrak{M} \subseteq A$. Following [20, §3.6], for every matrix $H \in M_{n \times n}(\mathfrak{A})$ there is a generalised adjoint matrix $H^{*} \in M_{n \times n}(\mathfrak{M})$ such that $H^{*} H=$ $H H^{*}=\operatorname{nr}(H) \cdot 1_{n \times n}$ (note that the conventions in [20, §3.6] slightly differ from those in [26]). If $\tilde{H} \in M_{n \times n}(\mathfrak{A})$ is a second matrix, then $(H \tilde{H})^{*}=\tilde{H}^{*} H^{*}$. We define

$$
\begin{aligned}
\mathcal{H}(\mathfrak{A}) & :=\left\{x \in \zeta(\mathfrak{A}) \mid x H^{*} \in M_{n \times n}(\mathfrak{A}) \forall H \in M_{n \times n}(\mathfrak{A}) \forall n \in \mathbb{N}\right\}, \\
\mathcal{I}(\mathfrak{A}) & :=\left\langle\operatorname{nr}(H) \mid H \in M_{n \times n}(\mathfrak{A}), n \in \mathbb{N}\right\rangle_{\zeta(\mathfrak{A})} .
\end{aligned}
$$

One can show that these are $R$-lattices satisfying

$$
\mathcal{H}(\mathfrak{A}) \cdot \mathcal{I}(\mathfrak{A})=\mathcal{H}(\mathfrak{A}) \subseteq \zeta(\mathfrak{A}) \subseteq \mathcal{I}(\mathfrak{A}) \subseteq \zeta(\mathfrak{M}) .
$$

Hence $\mathcal{H}(\mathfrak{A})$ is an ideal in the commutative $R$-order $\mathcal{I}(\mathfrak{A})$. We will refer to $\mathcal{H}(\mathfrak{A})$ and $\mathcal{I}(\mathfrak{A})$ as the denominator ideal and the integrality ring of the $R$-order $\mathfrak{A}$, respectively.

Remark 3.2. The integrality ring is the smallest subring of $\zeta(A)$ that contains $\zeta(\mathfrak{A})$ and the image of the reduced norm of all matrices with entries in $\mathfrak{A}$. The denominator ideal measures the failure of the generalised adjoint matrices having coefficients in $\mathfrak{A}$.

If $p$ is a prime and $G$ is a finite group, we set

$$
\begin{aligned}
\mathcal{I}(G) & :=\mathcal{I}(\mathbb{Z}[G]), & \mathcal{I}_{p}(G) & :=\mathcal{I}\left(\mathbb{Z}_{p}[G]\right), \\
\mathcal{H}(G) & :=\mathcal{H}(\mathbb{Z}[G]), & \mathcal{H}_{p}(G) & :=\mathcal{H}\left(\mathbb{Z}_{p}[G]\right) .
\end{aligned}
$$

The first claim of the following result is a special case of [20, Prop. 4.4]. The second claim then follows easily from (3.1). 
Proposition 3.3. Let $p$ be prime and $G$ be a finite group. Then, one has $\mathcal{H}_{p}(G)=$ $\zeta\left(\mathbb{Z}_{p}[G]\right)$ if and only if $p$ does not divide the order of the commutator subgroup of $G$. Moreover, in this case, we have $\mathcal{I}_{p}(G)=\zeta\left(\mathbb{Z}_{p}[G]\right)$.

Let $\mathfrak{A}=R[G]$, where $R$ is either $\mathbb{Z}$ or $\mathbb{Z}_{p}$ for a prime $p$. As above let $\mathfrak{M}$ be a maximal order containing $\mathfrak{A}$. The central conductor of $\mathfrak{M}$ over $\mathfrak{A}$ is defined to be $\mathcal{F}(\mathfrak{A}):=\{x \in \zeta(\mathfrak{M}) \mid x \mathfrak{M} \subseteq \mathfrak{A}\}$ and is explicitly given by (cf. [10, Thm. 27.13])

$$
\mathcal{F}(\mathfrak{A})=\bigoplus_{\chi} \frac{|G|}{\chi(1)} \mathcal{D}^{-1}(E(\chi) / E),
$$

where $\mathcal{D}^{-1}(E(\chi) / E)$ denotes the inverse different of the extension

$$
E(\chi):=E(\chi(g): g \in G) \text { over } E=\operatorname{Quot}(R)
$$

and the sum runs over all irreducible characters of $G$ modulo Galois action. It is clear from the definition that we always have $\mathcal{F}(\mathfrak{A}) \subseteq \mathcal{H}(\mathfrak{A})$. As above we set

$$
\mathcal{F}(G):=\mathcal{F}(\mathbb{Z}[G]), \quad \mathcal{F}_{p}(G):=\mathcal{F}\left(\mathbb{Z}_{p}[G]\right) .
$$

Example 3.4. Let $p$ and $\ell$ be primes with $\ell$ odd. We compute the denominator ideals of $\mathbb{Z}_{p}\left[D_{2 \ell}\right]$, where $D_{2 \ell}$ denotes the dihedral group of order $2 \ell$. In the case $\ell=3$, one has $D_{6} \simeq S_{3}$, the symmetric group on three letters. We let $\mathfrak{M}_{p}\left(D_{2 \ell}\right)$ be a maximal $\mathbb{Z}_{p}$-order containing $\mathbb{Z}_{p}\left[D_{2 \ell}\right]$. Then

$$
\begin{aligned}
& \mathcal{H}_{p}\left(D_{2 \ell}\right)= \begin{cases}\zeta\left(\mathbb{Z}_{p}\left[D_{2 \ell}\right]\right), & \text { if } p \neq \ell, \\
\mathcal{F}_{p}\left(D_{2 \ell}\right), & \text { if } p=\ell ;\end{cases} \\
& \mathcal{I}_{p}\left(D_{2 \ell}\right)= \begin{cases}\zeta\left(\mathbb{Z}_{p}\left[D_{2 \ell}\right]\right), & \text { if } p \neq \ell, \\
\zeta\left(\mathfrak{M}_{p}\left(D_{2 \ell}\right)\right) & \text { if } p=\ell .\end{cases}
\end{aligned}
$$

In fact, in the case that $p \neq \ell$, the result follows from Proposition 3.3 . In the case $p=\ell$, the result is established in [20, Ex. 6].

Example 3.5. Let $p$ be a prime and let $q=\ell^{n}$ be a prime power. We consider the group $\operatorname{Aff}(q)=\mathbb{F}_{q} \rtimes \mathbb{F}_{q}^{\times}$of affine transformations on $\mathbb{F}_{q}$, the finite field with $q$ elements. Let $\mathfrak{M}_{p}(\operatorname{Aff}(q))$ be a maximal $\mathbb{Z}_{p}$-order such that $\mathbb{Z}_{p}[\operatorname{Aff}(q)] \subseteq \mathfrak{M}_{p}(\operatorname{Aff}(q)) \subseteq \mathbb{Q}_{p}[\operatorname{Aff}(q)]$. Then by [21, Prop. 6.7] we have

$$
\begin{aligned}
& \mathcal{H}_{p}(\operatorname{Aff}(q))= \begin{cases}\zeta\left(\mathbb{Z}_{p}[\operatorname{Aff}(q)]\right), & \text { if } p \neq \ell, \\
\mathcal{F}_{p}(\operatorname{Aff}(q)), & \text { if } p=\ell \neq 2 ;\end{cases} \\
& \mathcal{I}_{p}(\operatorname{Aff}(q))= \begin{cases}\zeta\left(\mathbb{Z}_{p}[\operatorname{Aff}(q)]\right), & \text { if } p \neq \ell, \\
\zeta\left(\mathfrak{M}_{p}(\operatorname{Aff}(q))\right), & \text { if } p=\ell \neq 2 .\end{cases}
\end{aligned}
$$

If $p=\ell=2$, then we have containments

$$
\begin{aligned}
2 \mathcal{H}_{2}(\operatorname{Aff}(q)) & \subseteq \mathcal{F}_{2}(\operatorname{Aff}(q)) \subseteq \mathcal{H}_{2}(\operatorname{Aff}(q)), \\
2 \zeta\left(\mathfrak{M}_{2}(\operatorname{Aff}(q))\right) & \subseteq \mathcal{I}_{2}(\operatorname{Aff}(q)) \subseteq \zeta\left(\mathfrak{M}_{2}(\operatorname{Aff}(q))\right) .
\end{aligned}
$$

Note that the commutator subgroup of $\operatorname{Aff}(q)$ is $\mathbb{F}_{q}$ so that the case $p \neq \ell$ again follows from Proposition 3.3 . 
Example 3.6. Let $S_{4}$ be the symmetric group on 4 letters. If $p$ is an odd prime, then $\mathcal{I}_{p}\left(S_{4}\right)=\zeta\left(\mathfrak{M}_{p}\left(S_{4}\right)\right)$ and $\mathcal{H}_{p}\left(S_{4}\right)=\mathcal{F}_{p}\left(S_{4}\right)$. However, if $p=2$ we have

$$
\begin{aligned}
& \mathcal{F}_{2}\left(S_{4}\right) \subsetneq \mathcal{H}_{2}\left(S_{4}\right) \subsetneq \zeta\left(\mathbb{Z}_{2}\left[S_{4}\right]\right) ; \\
& \zeta\left(\mathbb{Z}_{2}\left[S_{4}\right]\right) \subsetneq \mathcal{I}_{2}\left(S_{4}\right) \subsetneq \zeta\left(\mathfrak{M}_{2}\left(S_{4}\right)\right) .
\end{aligned}
$$

This follows from [21, Prop. 6.8].

3.3. Integrality conjectures. Assume that $L / K$ is a Galois CM-extension with Galois group $G$. Let $S$ be a finite set of places of $K$ containing $S_{\text {ram }} \cup S_{\infty}$. We choose a maximal order $\mathfrak{M}(G)$ such that $\mathbb{Z}[G] \subseteq \mathfrak{M}(G) \subseteq \mathbb{Q}[G]$. If $T$ is a finite set of places of $K$ such that $\operatorname{Hyp}(S, T)$ is satisfied, then $\delta_{T}(0)=\operatorname{nr}\left(1-N(v) \phi_{w}^{-1}\right)$ belongs to $\zeta(\mathfrak{M}(G))$. We define $\mathfrak{A}_{S}$ to be the $\zeta(\mathbb{Z}[G])$-submodule of $\zeta(\mathfrak{M}(G))$ generated by the elements $\delta_{T}(0)$, where $T$ runs through the finite sets of places of $K$ such that $\operatorname{Hyp}(S, T)$ is satisfied.

Conjecture 3.7. Let $S$ be a finite set of places of $K$ containing $S_{\text {ram }} \cup S_{\infty}$. Then, we have an inclusion

$$
\mathfrak{A}_{S} \theta_{S} \subseteq \mathcal{I}(G) .
$$

As the integrality ring $\mathcal{I}(G)$ is always contained in the centre of the maximal order $\mathfrak{M}(G)$, we may also state the following considerably weaker conjecture.

Conjecture 3.8. Let $S$ be a finite set of places of $K$ containing $S_{\text {ram }} \cup S_{\infty}$. Then we have an inclusion

$$
\mathfrak{A}_{S} \theta_{S} \subseteq \zeta(\mathfrak{M}(G))
$$

Remark 3.9. If $p$ is a prime, we let $\mathfrak{M}_{p}(G):=\mathbb{Z}_{p} \otimes \mathfrak{M}(G)$ which is a maximal $\mathbb{Z}_{p}$-order in $\mathbb{Q}_{p}[G]$. As we have

$$
\mathcal{I}(G)=\zeta(\mathbb{Q}[G]) \cap \bigcap_{p} \mathcal{I}_{p}(G) \text { and } \zeta(\mathfrak{M}(G))=\zeta(\mathbb{Q}[G]) \cap \bigcap_{p} \zeta\left(\mathfrak{M}_{p}(G)\right)
$$

the integrality conjectures of this section naturally decompose into local conjectures at each prime $p$.

Example 3.10. Consider the Galois CM-extension $L / \mathbb{Q}$ from Example 3.1. As before let $S=S_{\text {ram }} \cup S_{\infty}$ and $T=\{7\}$. We have seen that in this case $\theta_{S}^{T}$ does not lie in $\zeta\left(\mathbb{Z}_{3}[G]\right)$. However, one has

$$
\theta_{S}^{T}=\operatorname{nr}\left((1-j)\left(-\frac{71}{2}+\frac{1}{2} \sigma-11 \sigma^{2}+19 \tau+\frac{13}{2} \sigma \tau+\frac{37}{2} \sigma^{2} \tau\right)\right) \in \mathcal{I}_{3}(G) .
$$

Theorem 3.11. Conjecture 3.7 and Conjecture 3.8 both hold when $L / K$ is abelian.

Proof. Lemma 2.8 implies that $\mathfrak{A}_{S}=\operatorname{Ann}_{\mathbb{Z}[G]}\left(\mu_{L}\right)$. Then, the result follows from (2.1) and the fact that $\mathcal{I}(G)=\mathbb{Z}[G]$ in this case.

Recall that a finite group is called monomial if each of its irreducible characters is induced by a linear character of a subgroup. The class of monomial groups includes all nilpotent groups [10, Thm. 11.3] and, more generally, all supersoluble groups [38, Ch. 2, Cor. 3.5].

Theorem 3.12. Let $L / K$ be a Galois extension with Galois group $G \simeq H \times C$, where $H$ is monomial and $C$ is abelian. Let $S$ be a finite set of places of $K$ containing $S_{\mathrm{ram}} \cup S_{\infty}$. Then, we have an inclusion

$$
\mathfrak{A}_{S} \theta_{S} \subseteq \zeta(\mathfrak{M}(H))[C]
$$

In particular, Conjecture 3.8 is true for monomial extensions. 
Proof. This is due to the author [30, Thm. 1.2]. The proof heavily relies on the abelian case and functoriality of Artin $L$-functions.

For non-abelian extensions, unconditional results on Conjecture 3.7 are rather sparse. Here we only mention the following special case of [30, Cor. 5.12].

Corollary 3.13. Let $\ell$ be an odd prime. Let $L / K$ be a Galois CM-extension with Galois group isomorphic to $D_{4 \ell}$, the dihedral group of order $4 \ell$. Then, Conjecture 3.7 holds.

Proof. We first note that $D_{4 \ell} \simeq D_{2 \ell} \times C_{2}$ with $C_{2}:=\mathbb{Z} / 2 \mathbb{Z}$ and that dihedral groups are monomial. Taking Example 3.4 into account, we see that the $p$-part of Conjecture 3.7 directly follows from Theorem 3.12 if $p$ is odd. Now consider the case $p=2$. Let $N$ be the commutator subgroup of $D_{2 \ell}$ so that $D_{2 \ell} / N \simeq C_{2}$. It follows from [21, Proposition 2.13] that the group ring $\mathbb{Z}_{2}\left[D_{2 \ell}\right]$ is ' $N$-hybrid' meaning that it decomposes into a direct product of $\mathbb{Z}_{2}\left[D_{2 \ell} / N\right] \simeq \mathbb{Z}_{2}\left[C_{2}\right]$ and some maximal order (see Definition 4.23 below). As

$$
\mathcal{I}_{2}\left(D_{4 \ell}\right)=\zeta\left(\mathbb{Z}_{2}\left[D_{4 \ell}\right]\right)=\zeta\left(\mathbb{Z}_{2}\left[D_{2 \ell}\right]\right)\left[C_{2}\right],
$$

the result follows by combining Theorems 3.12 and 3.11 .

We put $\omega_{L}:=\operatorname{nr}\left(\left|\mu_{L}\right|\right)=\operatorname{nr}\left(w_{L}\right)$.

Proposition 3.14. Let $S$ be a finite set of places of $K$ containing $S_{\text {ram }} \cup S_{\infty}$.

(i) Suppose that Conjecture 3.7 holds. Then,

$$
\omega_{L} \theta_{S} \in \mathcal{I}(G)
$$

(ii) Suppose that Conjecture 3.8 holds. Then,

$$
\omega_{L} \theta_{S} \in \zeta(\mathfrak{M}(G))
$$

Proof. It suffices to show that $\omega_{L} \theta_{S} \in \mathcal{A}_{p}(G)$ for each prime $p$, where $\mathcal{A}_{p}(G)=\mathcal{I}_{p}(G)$ in case (1) and $\mathcal{A}_{p}(G)=\zeta\left(\mathfrak{M}_{p}(G)\right)$ in case (2). By Lemma 2.8, there is a totally decomposed place $v_{0}$ of $K$ (in fact infinitely many places) such that $\left|\mu_{L}\right|=\left(1-N\left(v_{0}\right)\right) \cdot c$, where $c$ is a unit in $\mathbb{Z}_{p}$, and such that $\operatorname{Hyp}\left(S, T_{0}\right)$ is satisfied with $T_{0}:=\left\{v_{0}\right\}$. As $\operatorname{nr}(c)$ belongs to $\mathcal{A}_{p}(G)$, we have

$$
\omega_{L} \theta_{S}=\operatorname{nr}(c) \theta_{S}^{T_{0}} \in \mathcal{A}_{p}(G)
$$

as desired.

3.4. The non-abelian Brumer and Brumer-Stark conjectures. The following conjecture was first formulated in [27] and is a non-abelian generalisation of Brumer's Conjecture 2.1.

Conjecture $3.15(B(L / K, S))$. Let $S$ be a finite set of places of $K$ containing $S_{\mathrm{ram}} \cup S_{\infty}$. Then, for each $x \in \mathcal{H}(G)$, we have

$$
x \cdot \mathfrak{A}_{S} \theta_{S} \subseteq \operatorname{Ann}_{\zeta(\mathbb{Z}[G])}\left(\mathrm{cl}_{L}\right) .
$$

Remark 3.16. Suppose that $G$ is abelian. As we have already observed, Lemma 2.8 implies that $\mathfrak{A}_{S}=\operatorname{Ann}_{\mathbb{Z}[G]}\left(\mu_{L}\right)$ in this case. Since we have $\mathcal{H}(G)=\mathbb{Z}[G]$, Conjecture 3.15 recovers Brumer's Conjecture 2.1.

Remark 3.17. When Conjecture 3.7 holds, then $x \cdot \mathfrak{A}_{S} \theta_{S}$ is at least contained in $\zeta(\mathbb{Z}[G])$ for each $x \in \mathcal{H}(G)$. 
Remark 3.18. If $M$ is a finitely generated $\mathbb{Z}$-module and $p$ is a prime, we define its $p$ part to be $M(p):=\mathbb{Z}_{p} \otimes M$. Replacing the class group $\mathrm{cl}_{L}$ by $\mathrm{cl}_{L}(p)$ for each prime $p$, Conjecture $B(L / K, S)$ naturally decomposes into local conjectures $B(L / K, S, p)$. It is then possible to replace $\mathcal{H}(G)$ by $\mathcal{H}_{p}(G)$ (see [27, Lemma 1.4]). Moreover, if $p$ does not divide the order of the commutator subgroup of $G$ then $\mathcal{H}_{p}(G)=\mathcal{I}_{p}(G)=\zeta\left(\mathbb{Z}_{p}[G]\right)$ by Proposition 3.3 and so granting the hypotheses on $S$ the statement of the local conjecture simplifies to

$$
\mathfrak{A}_{S} \theta_{S} \subseteq \operatorname{Ann}_{\zeta\left(\mathbb{Z}_{p}[G]\right)}\left(\mathrm{cl}_{L}(p)\right) .
$$

Remark 3.19. Burns [6] has also formulated a conjecture which generalises many refined Stark conjectures to the non-abelian situation. In particular, it implies Conjecture 3.15 (see [6, Prop. 3.5.1]). A further approach to non-abelian Brumer and Brumer-Stark conjectures is due to Dejou and Roblot [14].

Recall that $\omega_{L}:=\operatorname{nr}\left(\left|\mu_{L}\right|\right)$. The following is a non-abelian generalisation of the BrumerStark Conjecture 2.5.

Conjecture $3.20(B S(L / K, S))$. Let $S$ be a finite set of places of $K$ containing $S_{\mathrm{ram}} \cup S_{\infty}$. Then for each $x \in \mathcal{H}(G)$ we have $x \cdot \omega_{L} \cdot \theta_{S} \in \zeta(\mathbb{Z}[G])$. Moreover, for each non-zero fractional ideal $\mathfrak{a}$ of $L$, there is an anti-unit $\epsilon=\epsilon(x, \mathfrak{a}, S) \in L^{\times}$such that

$$
\mathfrak{a}^{x \cdot \omega_{L} \cdot \theta_{S}}=(\epsilon)
$$

and for each finite set $T$ of primes of $K$ such that $\operatorname{Hyp}\left(S \cup S_{\epsilon}, T\right)$ is satisfied there is an $\epsilon_{T} \in E_{L, S_{\epsilon}}^{T}$ such that

for each $z \in \mathcal{H}(G)$.

$$
\epsilon^{z \cdot \delta_{T}(0)}=\epsilon_{T}^{z \cdot \omega_{L}}
$$

Remark 3.21. If $G$ is abelian, we have

$$
\mathcal{I}(G)=\mathcal{H}(G)=\mathbb{Z}[G] \quad \text { and } \quad \omega_{L}=\left|\mu_{L}\right|=w_{L} .
$$

Hence it suffices to treat the case $x=z=1$ in this situation. Then, Proposition 2.10 implies that Conjecture 3.20 generalises Conjecture 2.5 .

Remark 3.22. Suppose that Conjecture 3.7 holds. Then, $\omega_{L} \theta_{S} \in \mathcal{I}(G)$ by Proposition 3.14 and thus $x \cdot \omega_{L} \cdot \theta_{S} \in \zeta(\mathbb{Z}[G])$ for each $x \in \mathcal{H}(G)$.

Remark 3.23. When we restrict to ideals whose classes in $\mathrm{cl}_{L}$ have $p$-power order, we again obtain local conjectures $B S(L / K, S, p)$ for each prime $p$.

3.5. The weak Brumer and Brumer-Stark conjectures. Since $\mathcal{H}(G)$ always contains the central conductor $\mathcal{F}(G)$, we can state the following weaker versions of Conjectures $B(L / K, S)$ and $B S(L / K, S)$

Conjecture $3.24\left(B_{w}(L / K, S)\right)$. Let $S$ be a finite set of places of $K$ containing $S_{\mathrm{ram}} \cup S_{\infty}$. Then, for each $x \in \mathcal{F}(G)$ we have

$$
x \cdot \mathfrak{A}_{S} \theta_{S} \subseteq \operatorname{Ann}_{\zeta(\mathbb{Z}[G])}\left(\mathrm{cl}_{L}\right) .
$$

Conjecture $3.25\left(B S_{w}(L / K, S)\right)$. Let $S$ be a finite set of places of $K$ containing $S_{\text {ram }} \cup$ $S_{\infty}$. Then for each $x \in \mathcal{F}(G)$ we have $x \cdot \omega_{L} \cdot \theta_{S} \in \zeta(\mathbb{Z}[G])$. Moreover, for each non-zero fractional ideal $\mathfrak{a}$ of $L$, there is an anti-unit $\epsilon=\epsilon(x, \mathfrak{a}, S) \in L^{\times}$such that

$$
\mathfrak{a}^{x \cdot \omega_{L} \cdot \theta_{S}}=(\epsilon)
$$


and, for each finite set $T$ of primes of $K$ such that $\operatorname{Hyp}\left(S \cup S_{\epsilon}, T\right)$ holds, there is an $\epsilon_{T} \in E_{S_{\epsilon}}^{T}$ such that

$$
\epsilon^{z \cdot \delta_{T}(0)}=\epsilon_{T}^{z \cdot \omega_{L}}
$$

for each $z \in \mathcal{F}(G)$.

Remark 3.26. Suppose that Conjecture 3.8 holds. Then $x \cdot \mathfrak{A}_{S} \theta_{S}$ and $x \cdot \omega_{L} \cdot \theta_{S}$ both lie in $\zeta(\mathbb{Z}[G]$ ) (for the latter use Proposition 3.14).

Remark 3.27. We may again formulate local conjectures $B_{w}(L / K, S, p)$ and $B S_{w}(L / K, S, p)$ for each prime $p$.

3.6. Implications between the conjectures. We first discuss dependence on the set $S$.

Lemma 3.28. Let $S$ and $S^{\prime}$ be two finite sets of places of $K$ such that $S$ contains $S_{\text {ram }} \cup S_{\infty}$. If $S \subseteq S^{\prime}$, one has

$$
\begin{aligned}
B(L / K, S) & \Longrightarrow B\left(L / K, S^{\prime}\right) \\
B_{w}(L / K, S) & \Longrightarrow B_{w}\left(L / K, S^{\prime}\right) \\
B S(L / K, S) & \Longrightarrow B S\left(L / K, S^{\prime}\right) \\
B S_{w}(L / K, S) & \Longrightarrow B S_{w}\left(L / K, S^{\prime}\right) .
\end{aligned}
$$

Proof. We only give the proof in the case of Brumer's conjecture; the other cases follow similarly. So assume that $B(L / K, S)$ holds. We have $\theta_{S^{\prime}}=\operatorname{nr}(\lambda) \theta_{S}$, where $\lambda=$ $\prod_{v \in S^{\prime} \backslash S}\left(1-\phi_{w}^{-1}\right) \in \mathbb{Z}[G]$. If $x$ lies in $\mathcal{H}(G)$, so does $\tilde{x}:=x \cdot \operatorname{nr}(\lambda)$. Hence we see that $x \mathfrak{A}_{S^{\prime}} \theta_{S^{\prime}} \subseteq \tilde{x} \mathfrak{A}_{S} \theta_{S}$ belongs to $\zeta(\mathbb{Z}[G])$ and annihilates $\mathrm{cl}_{L}$.

The relation between the Brumer-Stark conjecture and Brumer's conjecture is slightly more subtle.

Lemma 3.29. Let $S$ be a finite set of places of $K$ containing $S_{\text {ram }} \cup S_{\infty}$. Then,

$$
\begin{aligned}
B S(L / K, S) & \Longrightarrow B(L / K, S) \\
B S_{w}(L / K, S) & \Longrightarrow B_{w}(L / K, S)
\end{aligned}
$$

Proof. We give the proof for the strong conjectures. Let $\mathfrak{a}$ be a non-zero fractional ideal of $L$ and let $x \in \mathcal{H}(G)$. Then $\mathfrak{a}^{x \cdot \omega_{L} \theta_{S}}=(\epsilon)$ and $(\epsilon)^{z \cdot \delta_{T}(0)}=\left(\epsilon_{T}\right)^{z \cdot \omega_{L}}$ for all $z \in \mathcal{H}(G)$. Hence

$$
\mathfrak{a}^{x \cdot z \cdot \omega_{L} \cdot \theta_{S}^{T}}=(\epsilon)^{z \cdot \delta_{T}(0)}=\left(\epsilon_{T}\right)^{z \cdot \omega_{L}} .
$$

Since $\omega_{L} \in \zeta(\mathbb{Q} G)^{\times}$, we find $N \in \mathbb{N}$ such that $N \cdot \omega_{L}^{-1} \in \zeta(\mathbb{Z}[G])$. Moreover, $|G| \cdot \zeta(\mathbb{Z}[G]) \subseteq$ $\mathcal{F}(G) \subseteq \mathcal{H}(G)$ such that we may choose $z=|G| \cdot N \cdot \omega_{L}^{-1}$. However, the group of fractional ideals has no $\mathbb{Z}$-torsion such that equation $(3.3)$ implies that $x \cdot \theta_{S}^{T}$ belongs to $\zeta(\mathbb{Z}[G])$ (take $\mathfrak{a}$ to be a totally decomposed prime) and $\mathfrak{a}^{x \cdot \theta_{S}^{T}}=\left(\epsilon_{T}\right)$.

\subsection{A strong Brumer-Stark property.}

Definition 3.30. Let $S$ be a finite set of places of $K$ containing $S_{\text {ram }} \cup S_{\infty}$. We say that $L / K$ satisfies the strong Brumer-Stark property $S B S(L / K, S)$ if

$$
\mathcal{H}(G) \cdot \frac{1}{2} \cdot \theta_{S}^{T} \subseteq \operatorname{Ann}_{\zeta(\mathbb{Z}[G])}\left(\mathrm{cl}_{L}^{T}\right)
$$

for all finite sets $T$ of $K$ such that $\operatorname{Hyp}(S, T)$ holds. 
Remark 3.31. It is clear that $S B S(L / K, S)$ holds if and only if

$$
\mathcal{H}_{p}(G) \cdot \frac{1}{2} \cdot \theta_{S}^{T} \subseteq \operatorname{Ann}_{\zeta\left(\mathbb{Z}_{p}[G]\right)}\left(\mathrm{cl}_{L}^{T}(p)\right)
$$

for all primes $p$. It is then not hard to show that the strong Brumer-Stark property $S t B S(L / K, S, p)$ as discussed in [27] for all $p$ implies our strong Brumer-Stark property $S B S(L / K, S)$.

Remark 3.32. Suppose that $S^{\prime}$ is a finite set of places of $K$ containing $S$. Then it follows as in the proof of Lemma 3.28 that $S B S(L / K, S)$ implies $S B S\left(L / K, S^{\prime}\right)$.

Proposition 3.33. Let $S$ be a finite set of places of $K$ containing $S_{\text {ram }} \cup S_{\infty}$. Then, $S B S(L / K, S)$ implies $B S(L / K, S)$.

Proof. The proof is similar to [27, Prop. 3.9].

Remark 3.34. In order to prove $B S(L / K, S)$ and $B(L / K, S)$, it thus suffices to show that, for every prime $p$, we have:

$$
\mathcal{H}_{p}(G) \cdot \frac{1}{2} \cdot \theta_{S}^{T} \subseteq \operatorname{Ann}_{\zeta\left(\mathbb{Z}_{p}[G]\right)}\left(\mathrm{cl}_{L}^{T}(p)\right)
$$

for all finite sets $T$ of $K$ such that $\operatorname{Hyp}(S, T)$ holds. We refer to this property as $S B S(L / K, S, p)$. This is in fact not much stronger than $B S(L / K, S, p)$. Nomura [32, Proposition 4.2] showed that for odd $p$ in fact $S B S(L / K, S, p)$ and $B S(L / K, S, p)$ are equivalent.

Remark 3.35. Replacing the denominator ideal $\mathcal{H}(G)$ by the central conductor $\mathcal{F}(G)$ one can formulate a weaker variant $S B S_{w}(L / K, S)$ such that $S B S_{w}(L / K, S)$ implies $B S_{w}(L / K, S)$ (we refrain from calling this the "weak strong Brumer-Stark property" for obvious reasons).

\section{Relations to FURTher CONJECTURES AND RESUlts}

4.1. The relation to the equivariant Tamagawa number conjecture. We only give a vague description of the statement of the equivariant Tamagawa number conjecture (ETNC) for the relevant Tate motive as formulated by Burns and Flach [3].

Let $L / K$ be a finite Galois extension of number fields with Galois group $G$. We regard $h^{0}(\operatorname{Spec}(L))$ as a motive defined over $K$ and with coefficients in the semisimple algebra $\mathbb{Q}[G]$. Let $\mathfrak{A}$ be a $\mathbb{Z}$-order such that $\mathbb{Z}[G] \subseteq \mathfrak{A} \subseteq \mathbb{Q}[G]$. The ETNC for the pair $\left(h^{0}(\operatorname{Spec}(L)), \mathfrak{A}\right)$ asserts that a certain canonical element $T \Omega(L / K, \mathfrak{A})$ of the relative algebraic $K$-group $K_{0}(\mathfrak{A}, \mathbb{R})$ vanishes. This element incorporates the leading coefficients of the Artin $L$-functions attached to the irreducible characters of $G$ and certain cohomological Euler characteristics.

We note that the ETNC for the pair $\left(h^{0}(\operatorname{Spec}(L)), \mathbb{Z}[G]\right)$, the Lifted Root Number Conjecture of Gruenberg, Ritter and Weiss [19], the vanishing of the element $T \Omega(L / K, 0)$ defined in [5, §2.1], and the 'leading term conjecture at $s=0$ ' of [2] are all equivalent (see [5, Theorems 2.3.3 and 2.4.1] and [2, Remarks 4.3 and 4.5]).

Moreover, Burns and Flach [4, §3, Cor. 1] have shown that the ETNC for the pair $\left(h^{0}(\operatorname{Spec}(L)), \mathfrak{M}(G)\right)$, where $\mathfrak{M}(G)$ is a maximal order, is equivalent to the strong Stark conjecture (as formulated by Chinburg [9, Conj. 2.2]) for $L / K$.

If $L / K$ is a Galois CM-extension, the ETNC (over the maximal order, and in general away from its 2-primary part) naturally decomposes into a plus and a minus part. The following result is due to the author [27, Thm. 3.5]. 
Theorem 4.1. Let $L / K$ be a Galois CM-extension of number fields with Galois group $G$. Let $\mathfrak{M}(G)$ be a maximal order in $\mathbb{Q}[G]$ containing $\mathbb{Z}[G]$. Then, the minus part of the ETNC for the pair $\left(h^{0}(\operatorname{Spec}(L)), \mathfrak{M}(G)\right)$ implies $B S_{w}(L / K, S)$ and $B_{w}(L / K, S)$ for all finite sets $S$ of places of $K$ containing $S_{\infty} \cup S_{\text {ram }}$.

There is also a prime-by-prime version of Theorem 4.1 (see [27, Thm. 4.1]). Combined with [28, Cor. 2] this leads to the following unconditional result (see also [27, Cor. 4.2]). We denote the maximal totally real subfield of $L$ by $L^{+}$and let $L^{\prime}$ be the Galois closure of $L$ over $\mathbb{Q}$. For a natural number $n$ we let $\zeta_{n}$ be a primitive $n$-th root of unity.

Theorem 4.2. Let $p$ be an odd prime. Assume that no prime of $L^{+}$above $p$ splits in $L$ whenever $L^{\prime} \subseteq\left(L^{\prime}\right)^{+}\left(\zeta_{p}\right)$. Then, $B S_{w}(L / K, S, p)$ and $B_{w}(L / K, S, p)$ are true for every set $S$ of places of $K$ containing $S_{\text {ram }} \cup S_{\infty}$.

Remark 4.3. We stress that for a given extension $L / K$ the hypotheses on $p$ in Theorem 4.2 are fulfilled by all primes that do not ramify in $L^{\prime}$. In particular, the hypotheses are satisfied by all but finitely many primes.

Theorem 4.4. Let $L / K$ be a Galois $C M$-extension of number fields with Galois group $G$. Let $p$ be an odd prime. Then, the minus p-part of the ETNC for the pair $\left(h^{0}(\operatorname{Spec}(L)), \mathbb{Z}[G]\right)$ implies $B S(L / K, S, p)$ and $B(L / K, S, p)$ for all finite sets $S$ of places of $K$ containing $S_{\infty} \cup S_{\text {ram }}$

Proof. When $\mu_{L}(p)$ is a cohomologically trivial $G$-module, this is due to Greither [17] (if $G$ is abelian) and to the author [27, Thm. 5.1]; this includes the cases $\mu_{L}(p)=1$ and $p \nmid|G|$. The general case follows from recent work of Burns [7, Proof of Cor. 3.11 (iii)].

Remark 4.5. The proofs of Theorem 4.4 actually show that a refinement of $S B S(L / K, S, p)$ holds and then use an argument similar to Proposition 3.33 .

There are meanwhile quite a few cases where the ETNC has been verified for certain non-abelian extensions. Here we only mention the following result of Johnston and the author [21, Thm. 4.6].

Theorem 4.6. Let $L / \mathbb{Q}$ be a Galois extension with Galois group $G \simeq \operatorname{Aff}(q)$, where $q=\ell^{n}$ is a prime power. Then, the ETNC for the pair $\left(h^{0}(\operatorname{Spec}(L)), \mathfrak{M}(G)\right)$ holds and the p-part of the ETNC for the pair $\left(h^{0}(\operatorname{Spec}(L)), \mathbb{Z}[G]\right)$ holds for every prime $p \neq \ell$.

Remark 4.7. An extension $L / \mathbb{Q}$ as in Theorem 4.6 never happens to be a CM-extension. However, Burns' conjecture on the annihilation of class groups [6] predicts non-trivial annihilators for any Galois extension of number fields. Theorem 4.6 can then be combined with Example 3.5 to show that Burns' conjecture holds in this case (up to a factor 2 if $\ell=2)$. This is [21, Thm. 7.6].

Remark 4.8. The $\ell$-part of the ETNC in the situation of Theorem 4.6 is considered in recent work with Henri Johnston [24. Suppose in addition that $L$ is totally real and Leopoldt's conjecture holds for $L$ at $\ell$. Then, the ETNC for the pair $\left(h^{0}(\operatorname{Spec}(L))(1), \mathbb{Z}[G]\right)$ holds. Moreover, the ETNC for the pair $\left(h^{0}(\operatorname{Spec}(L)), \mathbb{Z}[G]\right)$ holds if $\ell$ is at most tamely ramified (see [24, Cor. 10.6]). For the proof one has to verify the ' $\ell$-adic Stark conjecture at $s=1$ ' for $L / \mathbb{Q}$ which might be seen as an analogue at $s=1$ of Gross' conjecture 4.15 below. 
4.2. $p$-adic Artin $L$-functions. Let $p$ be an odd prime and let $K$ be a totally real field. Let $\mathcal{L} / K$ be a Galois extension of $K$ such that $\mathcal{L}$ is totally real and contains the cyclotomic $\mathbb{Z}_{p}$-extension $K_{\infty}$ of $K$ and $\left[\mathcal{L}: K_{\infty}\right]$ is finite. We put $\mathcal{G}:=\operatorname{Gal}(\mathcal{L} / K)$ and $\Gamma_{K}:=\operatorname{Gal}\left(K_{\infty} / K\right) \simeq \mathbb{Z}_{p}$ such that $\mathcal{G} \simeq H \rtimes \Gamma$, where $H:=\operatorname{Gal}\left(\mathcal{L} / K_{\infty}\right)$ and $\Gamma=\mathcal{G} / H \simeq \Gamma_{K}$. Thus $\mathcal{L} / K$ is a one-dimensional $p$-adic Lie extension. We choose a topological generator $\gamma_{K}$ of $\Gamma_{K}$.

We write $\chi_{\text {cyc }}$ for the $p$-adic cyclotomic character

$$
\chi_{\text {cyc }}: \operatorname{Gal}\left(\mathcal{L}\left(\zeta_{p}\right) / K\right) \longrightarrow \mathbb{Z}_{p}^{\times}
$$

defined by $\sigma(\zeta)=\zeta^{\chi_{\text {cyc }}(\sigma)}$ for any $\sigma \in \operatorname{Gal}\left(\mathcal{L}\left(\zeta_{p}\right) / K\right)$ and any $p$-power root of unity $\zeta$. Let $\omega$ and $\kappa$ denote the composition of $\chi_{\text {cyc }}$ with the projections onto the first and second factors of the canonical decomposition $\mathbb{Z}_{p}^{\times}=\mu_{\mathbb{Q}_{p}} \times\left(1+p \mathbb{Z}_{p}\right)$, respectively; thus $\omega$ is the Teichmüller character. We note that $\kappa$ factors through $\Gamma_{K}$ and put $u:=\kappa\left(\gamma_{K}\right)$.

Fix a character $\psi \in \operatorname{Irr}_{\mathbb{C}_{p}}(\mathcal{G})$ and let $S$ be a finite set of places of $K$ containing all archimedean places and all places that ramify in $\mathcal{L} / K$. Note that $S$ in particular contains the set $S_{p}$ of all $p$-adic places. Each topological generator $\gamma_{K}$ of $\Gamma_{K}$ permits the definition of a power series $G_{\psi, S}(T) \in \mathbb{Q}_{p}^{c} \otimes_{\mathbb{Q}_{p}} \operatorname{Quot}\left(\mathbb{Z}_{p}[[T]]\right)$ by starting out from the Deligne-Ribet power series for one-dimensional characters of open subgroups of $\mathcal{G}$ (see [8, 13, 1]) and then extending to the general case by using Brauer induction (see [16]). One then has an equality

$$
L_{p, S}(1-s, \psi)=\frac{G_{\psi, S}\left(u^{s}-1\right)}{H_{\psi}\left(u^{s}-1\right)},
$$

where $L_{p, S}(s, \psi): \mathbb{Z}_{p} \rightarrow \mathbb{C}_{p}$ denotes the 'S$S$-truncated $p$-adic Artin $L$-function' (a $p$-adic meromorphic function) attached to $\psi$ constructed by Greenberg [16], and where, for irreducible $\psi$, one has

$$
H_{\psi}(T)= \begin{cases}\psi\left(\gamma_{K}\right)(1+T)-1, & \text { if } H \subseteq \operatorname{ker} \psi, \\ 1, & \text { otherwise. }\end{cases}
$$

4.3. The interpolation property and Gross' conjecture. Let $p$ be an odd prime and choose a field isomorphism $\iota: \mathbb{C} \simeq \mathbb{C}_{p}$. For a character $\psi \in \operatorname{Irr}_{\mathbb{C}_{p}}(\mathcal{G})$ we put $\psi^{\iota}:=\iota^{-1} \circ \psi \in \operatorname{Irr}_{\mathbb{C}}(\mathcal{G})$. If $\psi$ is a linear character and $r \geq 1$ is an integer then for every choice of field isomorphism $\iota: \mathbb{C} \simeq \mathbb{C}_{p}$ one has the interpolation property

$$
L_{p, S}(1-r, \psi)=\iota\left(L_{S}\left(1-r,\left(\psi \omega^{-r}\right)^{\iota}\right)\right) .
$$

This can be extended to characters $\psi$ of arbitrary degree provided that $r \geq 2$ (see [16, $\S 4]$ ). However, the argument fails in the case $r=1$. Nevertheless, it seems plausible to conjecture the following.

Conjecture 4.9. For each $\psi \in \operatorname{Irr}_{\mathbb{C}_{p}}(\mathcal{G})$, one has

$$
L_{p, S}(0, \psi)=\iota\left(L_{S}\left(0,\left(\psi \omega^{-1}\right)^{\iota}\right)\right) .
$$

Remark 4.10. As both sides in (4.9) are well-behaved with respect to direct sum, inflation and induction of characters, it is easy to see that Conjecture 4.9 holds when $\psi$ is a monomial character (also see the discussion in [15, §2]).

In fact, Conjecture 4.9 is a special case of a Conjecture of Gross [15] which we now recall. Let $\chi \in \operatorname{Irr}_{\mathbb{C}}\left(\operatorname{Gal}\left(\mathcal{L}\left(\zeta_{p}\right) / K\right)\right)$ be a non-trivial character and let $r_{S}(\chi)$ be the order of vanishing of the $S$-truncated Artin $L$-function $L_{S}(s, \chi)$ at $s=0$. We write $L_{S}^{*}(0, \chi)$ 
for the leading coefficient in the Laurent series expansion of $L_{S}(s, \chi)$ at $s=0$. Now let $\psi \in \operatorname{Irr}_{\mathbb{C}_{p}}(\mathcal{G})$ and choose a field isomorphism $\iota: \mathbb{C} \simeq \mathbb{C}_{p}$. Then, formula (1.1) shows that

$$
r_{S}(\psi):=r_{S}\left(\left(\psi \omega^{-1}\right)^{\iota}\right)
$$

does in fact not depend on the choice of $\iota$. The first part of Gross' conjecture [15] concerns the order of vanishing of $p$-adic Artin $L$-functions and asserts the following.

Conjecture 4.11. Let $\psi \in \operatorname{Irr}_{\mathbb{C}_{p}}(\mathcal{G})$. Then, the order of vanishing of the S-truncated p-adic Artin L-function $L_{p, S}(s, \psi)$ at $s=0$ equals $r_{S}(\psi)$.

Remark 4.12. Suppose that $\psi$ is linear. If $r_{S}(\psi)$ vanishes, Conjecture 4.11 holds by (4.1). The conjecture is also known when $r_{S}(\psi)=1$ (see [15, Prop. 2.13]).

Theorem 4.13. Let $\psi \in \operatorname{Irr}_{\mathbb{C}_{p}}(\mathcal{G})$. Then, the order of vanishing of the $S$-truncated $p$-adic Artin L-function $L_{p, S}(s, \psi)$ at $s=0$ is at least $r_{S}(\psi)$.

Proof. For $\psi$ being a linear character, this has been proved by Spiess [35] using Shintani cocycles (his approach actually allows $p$ to be equal to 2). The general case has recently been settled by Burns [7, Thm. 3.1].

The rest of this subsection is mainly devoted to (the second part of) Gross' conjecture. This may be skipped by the reader who is only interested in the Brumer and BrumerStark conjectures.

Fix a character $\psi \in \operatorname{Irr}_{\mathbb{C}_{p}}(\mathcal{G})$ and choose a Galois CM-extension $L$ over $K$ such that $\psi \omega^{-1}$ factors through $G:=\operatorname{Gal}(L / K)$. We denote the kernel of the natural augmentation map $\mathbb{Z}[S(L)] \rightarrow \mathbb{Z}$ that maps each $w \in S(L)$ to 1 by $X_{L, S}$. The usual Dirichlet map

$$
\begin{aligned}
\lambda_{L, S}: \mathbb{R} \otimes E_{L, S} & \rightarrow \mathbb{R} \otimes X_{L, S} \\
1 \otimes \epsilon & \mapsto \sum_{w \in S(L)} \log |\epsilon|_{w} w
\end{aligned}
$$

is an isomorphism of $\mathbb{R}[G]$-modules. For each place $w$ of $L$, Gross [15, §1] defines a $p$-adic absolute value

as the composite map

$$
\|\cdot\|_{w, p}: L^{\times} \rightarrow \mathbb{Z}_{p}^{\times}
$$

$$
L^{\times} \hookrightarrow L_{w}^{\times} \rightarrow \operatorname{Gal}\left(L_{w}^{\mathrm{ab}} / L_{w}\right) \rightarrow \mathbb{Z}_{p}^{\times}
$$

here, $L_{w}^{\text {ab }}$ denotes the maximal abelian extension of $L_{w}$, the first arrow is the natural inclusion, the second arrow is the reciprocity map of local class field theory and the last map is the $p$-adic cyclotomic character. We define a homomorphism of $\mathbb{Z}_{p}[G]$-modules

$$
\begin{aligned}
\lambda_{p, L, S}: \mathbb{Z}_{p} \otimes E_{L, S} & \rightarrow \mathbb{Z}_{p} \otimes X_{L, S} \\
1 \otimes \epsilon & \mapsto \sum_{w \in S(L)} \log \|\epsilon\|_{w, p} w .
\end{aligned}
$$

Now, choose a field isomorphism $\iota: \mathbb{C} \simeq \mathbb{C}_{p}$. Then, $\lambda_{L, S}^{-1}$ and $\lambda_{p, L, S}$ induce an endomorphism

$$
\left(\mathbb{C}_{p} \otimes_{\mathbb{Z}_{p}} \lambda_{p, L, S}\right) \circ\left(\mathbb{C}_{p} \otimes_{\iota} \lambda_{L, S}^{-1}\right): \mathbb{C}_{p} \otimes X_{L, S} \rightarrow \mathbb{C}_{p} \otimes X_{L, S}
$$

We define a $p$-adic regulator

$$
R_{p, S}^{(\iota)}(\psi):=\operatorname{det}_{\mathbb{C}_{p}}\left(\left(\mathbb{C}_{p} \otimes_{\mathbb{Z}_{p}} \lambda_{p, L, S}\right) \circ\left(\mathbb{C}_{p} \otimes_{\iota} \lambda_{L, S}^{-1}\right) \mid \operatorname{Hom}_{\mathbb{C}_{p}[G]}\left(V_{\psi \omega^{-1}}, \mathbb{C}_{p} \otimes X_{L, S}\right)\right) .
$$

Proposition 4.14. Fix $\psi \in \operatorname{Irr}_{\mathbb{C}_{p}}(\mathcal{G})$ and choose a field isomorphism $\iota: \mathbb{C} \simeq \mathbb{C}_{p}$. Then Conjecture 4.11 holds for $\psi$ if and only if $R_{p, S}^{(\iota)}(\psi) \neq 0$. 
Proof. This follows from [7, Thm. 3.1 (iii)].

For an integer $r$, let $L_{p, S}^{r}(0, \psi)$ be the coefficient of $s^{r}$ in the power series expansion of $L_{p, S}(s, \psi)$ at $s=0$. We can now state the second part of Gross' conjecture.

Conjecture 4.15. Fix $\psi \in \operatorname{Irr}_{\mathbb{C}_{p}}(\mathcal{G})$ and choose a field isomorphism $\iota: \mathbb{C} \simeq \mathbb{C}_{p}$. Then, one has

$$
L_{p, S}^{r_{S}(\psi)}(0, \psi)=R_{p, S}^{(\iota)}(\psi) \cdot \iota\left(L_{S}^{*}\left(0,\left(\psi \omega^{-1}\right)^{\iota}\right)\right) .
$$

Remark 4.16. By means of Proposition 4.14, it is clear that Conjecture 4.15 is only interesting when Conjecture 4.11 holds.

The following recent result due to Dasgupta, Kakde and Ventullo [12] generalises the approach developed in [11].

Theorem 4.17. Conjecture 4.15 holds for linear characters.

Corollary 4.18. Suppose that Conjecture 4.11 holds for all $\psi \in \operatorname{Irr}_{\mathbb{C}_{p}}(\mathcal{G})$. Then, Conjecture 4.15 is also true for all $\psi \in \operatorname{Irr}_{\mathbb{C}_{p}}(\mathcal{G})$.

Proof. This follows from Theorem 4.17 by Brauer induction.

4.4. Conditional Results. We can now state the following result which has been proved by Johnston and the author [23, Thm. 5.2 and Cor. 5.4]. We refer to the 'equivariant Iwasawa main conjecture' (EIMC) for totally real fields (see [25] or [33], for instance).

Theorem 4.19. Let $L / K$ be a Galois $C M$-extension with Galois group $G$ and let $p$ be an odd prime. Suppose that the EIMC for the extension $L\left(\zeta_{p}\right)_{\infty}^{+} / K$ holds. Suppose further that Conjecture 4.9 holds for all irreducible characters of $\operatorname{Gal}\left(L\left(\zeta_{p}\right)_{\infty}^{+} / K\right)$ which factor through $\operatorname{Gal}\left(L^{+} / K\right)$. Then, $S B S(L / K, S, p)$ and thus $B S(L / K, S, p)$ and $B(L / K, S, p)$ are true for every finite set $S$ of places of $K$ containing $S_{p} \cup S_{\text {ram }} \cup S_{\infty}$.

Remark 4.20. We write $\mu_{p}(F)$ for the $p$-adic $\mu$-invariant attached to the cyclotomic $\mathbb{Z}_{p^{-}}$ extension of a number field $F$ (see [22, Rem. 4.3] for details). When $\mu_{p}\left(L\left(\zeta_{p}\right)^{+}\right)$vanishes, then the EIMC has been proved independently by Kakde [25] and Ritter and Weiss [33]. Without assuming the vanishing of $\mu$-invariants considerable progress has been made in [22]. This includes the case $p \nmid|G|$.

Remark 4.21. Suppose that $L / K$ is abelian. Then, Conjecture 4.9 holds by (4.1). Under the somewhat stronger condition that $\mu$ vanishes, Theorem 4.19 has been shown by Greither and Popescu [18] by an entirely different method. This method has been generalised to arbitrary Galois CM-extensions by the author in [29].

In order to get rid of the $p$-adic places one has to assume the full strength of Gross' conjecture.

Theorem 4.22. Let $L / K$ be a Galois CM-extension with Galois group $G$ and let $p$ be an odd prime. Suppose that $\mu_{p}\left(L^{+}\right)$vanishes or that $p \nmid|G|$. Suppose further that Gross' Conjecture 4.11 holds for all $\psi \in \operatorname{Irr}_{\mathbb{C}_{p}}\left(\operatorname{Gal}\left(L_{\infty}^{+} / K\right)\right)$. Then, the minus p-part of the ETNC for the pair $h^{0}(\operatorname{Spec}(L), \mathbb{Z}[G])$ holds. In particular, both $B S(L / K, S, p)$ and $B(L / K, S, p)$ are true for all finite sets $S$ of places of $K$ containing $S_{\text {ram }} \cup S_{\infty}$.

Proof. This is [7, Cors. 3.8 and 3.11]. Note that the last part follows from Theorem 4.4 . 
4.5. Unconditional results. We now discuss certain cases where the Brumer-Stark conjecture holds unconditionally. Let $p$ be a prime and let $G$ be a finite group. For a normal subgroup $N \unlhd G$, let $e_{N}=|N|^{-1} \sum_{\sigma \in N} \sigma$ be the associated central trace idempotent in the group algebra $\mathbb{Q}_{p}[G]$.

Definition 4.23. Let $N \unlhd G$. We say that the $p$-adic group ring $\mathbb{Z}_{p}[G]$ is $N$-hybrid if (i) $e_{N} \in \mathbb{Z}_{p}[G]$ (i.e. $p \nmid|N|$ ) and (ii) $\mathbb{Z}_{p}[G]\left(1-e_{N}\right)$ is a maximal $\mathbb{Z}_{p}$-order in $\mathbb{Q}_{p}[G]\left(1-e_{N}\right)$.

Theorem 4.24. Let $L / K$ be a finite Galois $C M$-extension of number fields. Let $N$ be a normal subgroup of $G:=\operatorname{Gal}\left(L^{+} / K\right)$ and let $F=\left(L^{+}\right)^{N}$. Let $p$ be an odd prime and let $\bar{P}$ be a Sylow p-subgroup of $\bar{G}:=\operatorname{Gal}(F / K) \simeq G / N$. Suppose that $\mathbb{Z}_{p}[G]$ is $N$-hybrid, $G$ is monomial, and $F^{\bar{P}} / \mathbb{Q}$ is abelian. Let $S$ be a finite set of places of $K$ such that $S_{p} \cup S_{\mathrm{ram}}(L / K) \cup S_{\infty} \subseteq S$. Then, both $B S(L / K, S, p)$ and $B(L / K, S, p)$ are true.

Proof. It follows from the theory of hybrid Iwasawa algebras [22] that the relevant case of the EIMC holds. We also recall that Conjecture 4.9 holds for monomial characters. Then the result follows from Theorem 4.19. See [23, Thm. 10.5] for details.

We recall that a Frobenius group is a finite group $G$ with a proper nontrivial subgroup $V$ such that $V \cap g V g^{-1}=\{1\}$ for all $g \in G-V$, in which case $V$ is called a Frobenius complement. A Frobenius group $G$ contains a unique normal subgroup $U$, known as the Frobenius kernel, such that $G$ is a semidirect product $U \rtimes V$.

Corollary 4.25. Let $L / K$ be a finite Galois CM-extension of number fields and let $G=\operatorname{Gal}\left(L^{+} / K\right)$. Suppose that $G=U \rtimes V$ is a Frobenius group with Frobenius kernel $U$ and abelian Frobenius complement $V$. Suppose further that $\left(L^{+}\right)^{U} / \mathbb{Q}$ is abelian (in particular, this is the case when $K=\mathbb{Q})$. Let $p$ be an odd prime and let $S$ be a finite set of places of $K$ such that $S_{p} \cup S_{\mathrm{ram}}(L / K) \cup S_{\infty} \subseteq S$. Suppose that either $p \nmid|U|$ or $U$ is a p-group (in particular, this is the case if $U$ is an $\ell$-group for any prime $\ell$.) Then, both $B S(L / K, S, p)$ and $B(L / K, S, p)$ are true.

Proof. This is [23, Cor. 10.7]. We recall the proof for convenience. First note that $G$ is monomial by [23, Lemma 9.7] since $V$ is abelian. Suppose that $p \nmid|U|$. Let $N=U$ and $F=\left(L^{+}\right)^{N}$. Then $\mathbb{Z}_{p}[G]$ is $N$-hybrid by [23, Prop. 9.4]. Hence, the desired result follows from Theorem 4.24 in this case since $F / \mathbb{Q}$ is abelian, which forces $F^{\bar{P}} / \mathbb{Q}$ to be abelian. Suppose that $U$ is a $p$-group. Taking $N=\{1\}$ and $F=L^{+}$, we apply Theorem 4.24 with $\bar{G}=G$ and $\bar{P}=U$ to obtain the desired result.

Example 4.26. In particular, $U$ is an $\ell$-group in Corollary 4.25 when $G$ is one of the following Frobenius groups (for a natural number $n$ we denote the cyclic group of order $n$ by $\left.C_{n}\right)$ :

- $G \simeq \operatorname{Aff}(q)=\mathbb{F}_{q} \rtimes \mathbb{F}_{q}^{\times}$, where $q$ is a prime power and $\operatorname{Aff}(q)$ is the group of affine transformations on $\mathbb{F}_{q}$,

- $G \simeq C_{\ell} \rtimes C_{q}$, where $q<\ell$ are distinct primes such that $q \mid(\ell-1)$ and $C_{q}$ acts on $C_{\ell}$ via an embedding $C_{q} \hookrightarrow \operatorname{Aut}\left(C_{\ell}\right)$,

- $G$ is isomorphic to any of the Frobenius groups constructed in [22, Ex. 2.11].

Note that in particular $\operatorname{Aff}(3) \simeq S_{3}$ is the symmetric group on 3 letters (which is the smallest non-abelian group) and $\operatorname{Aff}(4) \simeq A_{4}$ is the alternating group on 4 letters.

In certain situations, we can also remove the condition that $S_{p} \subseteq S$. To illustrate this, we conclude with the following two results (the first is [23, Thm. 10.10], whereas the second is due to Nomura [31, 32]). 
Theorem 4.27. Let $L / \mathbb{Q}$ be a finite Galois CM-extension of the rationals. Suppose that $\operatorname{Gal}(L / \mathbb{Q}) \simeq\langle j\rangle \times G$, where $G=\operatorname{Gal}\left(L^{+} / \mathbb{Q}\right)=N \rtimes V$ is a Frobenius group with Frobenius kernel $N$ and abelian Frobenius complement $V$. Suppose further that $N$ is an $\ell$-group for some prime $\ell$. Then, both $B S(L / \mathbb{Q}, S, p)$ and $B(L / \mathbb{Q}, S, p)$ are true for every odd prime $p$ and every finite set $S$ of places of $\mathbb{Q}$ such that $S_{\mathrm{ram}}(L / \mathbb{Q}) \cup S_{\infty} \subseteq S$.

Theorem 4.28. Let $\ell$ be an odd prime. Let $L / K$ be a Galois CM-extension with Galois group $G \simeq D_{4 \ell}$. Let $p$ be a prime and suppose that $p$ does not split in $\mathbb{Q}\left(\zeta_{\ell}\right)$. Then, $B S(L / K, S, p)$ and $B(L / K, S, p)$ both hold for every finite set $S$ of places of $K$ such that $S_{\text {ram }} \cup S_{\infty} \subseteq S$.

\section{REFERENCES}

[1] D. Barsky, Fonctions zeta $p$-adiques d'une classe de rayon des corps de nombres totalement réels. In Groupe d'Etude d'Analyse Ultramétrique (5e année: 1977/78), Secrétariat Math., Paris, 1978, Exp. No. 16 , pp. 1-23.

[2] D. Burns and M. Breuning, Leading terms of Artin $L$-functions at $s=0$ and $s=1$, Compos. Math. 143 (2007), 1427-1464.

[3] D. Burns and M. Flach, Tamagawa numbers for motives with (non-commutative) coefficients, Doc. Math. 6 (2001), 501-570.

[4] D. Burns and M. Flach, Tamagawa numbers for motives with (non-commutative) coefficients. II, Amer. J. Math. 125 (2003), 475-512.

[5] D. Burns, Equivariant Tamagawa numbers and Galois module theory. I, Compos. Math. 129 (2001), 203-237.

[6] D. Burns, On derivatives of Artin L-series, Invent. Math. 186 (2011), 291-371.

[7] D. Burns, On derivatives of $p$-adic $L$-series at $s=0$, preprint.

[8] P. Cassou-Noguès, Valeurs aux entiers négatifs des fonctions zêta et fonctions zêta $p$-adiques, Invent. Math. 51 (1979), 29-59.

[9] T. Chinburg, On the Galois structure of algebraic integers and S-units, Invent. Math. 74 (1983), 321-349.

[10] C.W. Curtis and I. Reiner, Methods of Representation Theory with Applications to Finite Groups and Orders, Vol. 1, Wiley, New York, 1981.

[11] S. Dasgupta, H. Darmon and R. Pollack, Hilbert modular forms and the Gross-Stark conjecture, Ann. of Math. 174 (2011), 439-484.

[12] S. Dasgupta, M. Kakde and K. Ventullo, On the Gross-Stark conjecture, preprint arXiv: 1605.08169.

[13] P. Deligne and K.A. Ribet, Values of abelian $L$-functions at negative integers over totally real fields, Invent. Math. 59 (1980), 227-286.

[14] G. Dejou and X.-F. Roblot, A Brumer-Stark conjecture for non-abelian extensions, J. Number Th. 142 (2014), 51-88.

[15] B.H. Gross, p-adic L-series at $s=0$, J. Fac. Sci. Univ. Tokyo Sect. IA Math. 28 (1981), 979-994.

[16] R. Greenberg, On p-adic Artin L-functions, Nagoya Math. J. 89 (1983), 77-87.

[17] C. Greither, Determining Fitting ideals of minus class groups via the equivariant Tamagawa number conjecture, Compos. Math. 143 (2007), 1399-1426.

[18] C. Greither and C.D. Popescu, An equivariant main conjecture in Iwasawa theory and applications, J. Algebraic Geom. 24 (2015), 629-692.

[19] K.W. Gruenberg, J. Ritter and A. Weiss, A local approach to Chinburg's root number conjecture, Proc. London Math. Soc. 79 (1999), 47-80.

[20] H. Johnston and A. Nickel, Noncommutative Fitting invariants and improved annihilation results, J. Lond. Math. Soc. 88 (2013), 137-160.

[21] H. Johnston and A. Nickel, On the equivariant Tamagawa number conjecture for Tate motives and unconditional annihilation results, Trans. Amer. Math. Soc. 368 (2016), 6539-6574. 
[22] H. Johnston and A. Nickel, Hybrid Iwasawa algebras and the equivariant Iwasawa main conjecture, Amer. J. Math. (to appear) arXiv:1408.4934.

[23] H. Johnston and A. Nickel, On the non-abelian Brumer-Stark conjecture, preprint arXiv: 1509.00200.

[24] H. Johnston and A. Nickel, On the $p$-adic Stark conjecture at $s=1$ and applications, preprint arXiv: 1703.06803.

[25] M. Kakde, The main conjecture of Iwasawa theory for totally real fields, Invent. Math. 193 (2013), 539-626.

[26] A. Nickel, Non-commutative Fitting invariants and annihilation of class groups, J. Algebra 323 (2010), 2756-2778

[27] A. Nickel, On non-abelian Stark-type conjectures, Ann. Inst. Fourier 61 (2011), 2577-2608.

[28] A. Nickel, On the equivariant Tamagawa number conjecture in tame CM-extensions, Math. Z. 268 (2011), 1-35.

[29] A. Nickel, Equivariant Iwasawa theory and non-abelian Stark-type conjectures, Proc. Lond. Math. Soc. 106 (2013), 1223-1247.

[30] A. Nickel, Integrality of Stickelberger elements and the equivariant Tamagawa number conjecture, J. Reine Angew. Math. (Crelle) $\mathbf{7 1 9}$ (2016), 101-132.

[31] J. Nomura, On non-abelian Brumer and Brumer-Stark conjecture for monomial CM-extensions, Int. J. Number Th. 10 (2014), 817-848.

[32] J. Nomura, The 2-part of the non-abelian Brumer-Stark conjecture for extensions with group $D_{4 p}$ and numerical examples of the conjecture. In Algebraic Number Theory and Related Topics 2012, RIMS Kôkyûroku Bessatsu, B51, Res. Inst. Math. Sci. (RIMS), Kyoto, 2014.

[33] J. Ritter and A. Weiss, On the "main conjecture" of equivariant Iwasawa theory, J. Amer. Math. Soc. 24 (2011), 1015-1050.

[34] C.L. Siegel, Über die Fourierschen Koeffizienten von Modulformen, Nachr. Akad. Wiss. Göttingen Math.-Phys. Kl. II 1970 (1970), 15-56.

[35] M. Spiess, Shintani cocycles and the order of vanishing of $p$-adic Hecke $L$-series at $s=0, M a t h$. Ann. 359 (2014), 239-265.

[36] L. Stickelberger, Ueber eine Verallgemeinerung der Kreistheilung, Math. Ann. 37 (1890), 321-367.

[37] J. Tate, Les conjectures de Stark sur les fonctions $L$ d'Artin en $s=0$, Lecture notes edited by D. Bernardi and N. Schappacher, Progress in Mathematics 47, Birkhäuser Boston, Boston, 1984.

[38] M. Weinstein (ed.), Between Nilpotent and Solvable, Polygonal Publ. House, Passaic, 1982.

Universität Duisburg-Essen, Fakultät für Mathematik, Thea-Leymann-Str. 9, 45127 Essen, Germany

E-mail address: andreas.nickel@uni-due.de

URL: https://www. uni-due.de/ hm0251/english.html 